Research Article

\title{
Effect of Activated Platelet-Rich Plasma on Chondrogenic Differentiation of Rabbit Bone Marrow-Derived Mesenchymal Stem Cells
}

\author{
Zhen Wang, ${ }^{1}$ Zheng Wang, ${ }^{2}$ Bin Zhang, ${ }^{1}$ Qinghua Zhao, ${ }^{1}$ Yubao Liu, ${ }^{1}$ and Wei Qi $\mathbb{D}^{2}$ \\ ${ }^{1}$ Department of Orthopedics, The First Hospital Affiliated to China Pharmaceutical University, Nanjing 211500, China \\ ${ }^{2}$ Department of Orthopedics, Zhenjiang Jinshan Hospital, Zhenjiang 212000, China \\ Correspondence should be addressed to Wei Qi; qiwei20153128@126.com
}

Received 29 March 2021; Revised 7 July 2021; Accepted 11 August 2021; Published 26 August 2021

Academic Editor: Elena A. Jones

Copyright (c) 2021 Zhen Wang et al. This is an open access article distributed under the Creative Commons Attribution License, which permits unrestricted use, distribution, and reproduction in any medium, provided the original work is properly cited.

\begin{abstract}
We aimed to evaluate the effect of activated platelet-rich plasma (PRP) on proliferation and chondrogenic differentiation of bone marrow-derived mesenchymal stem cells (BMSCs). Six mature male rabbits were included in this study. PRP was obtained by twostep centrifugation from whole blood, and it was activated using $\mathrm{CaCl}_{2}$ solution. BMSCs were isolated and proliferated from bone marrow of rabbits and characterized by flow cytometry. Passage 3 BMSCs were cultured in high-glucose Dulbecco's modified Eagle's medium (HG-DMEM) with the four different compositions for consecutive 7 days, including $10 \%$ fetal bovine serum, $5 \%$ PRP, 10\% PRP, and 15\% PRP. Cell counting assays were performed to evaluate the cell proliferation of BMSCs. BMSCs $\left(5 \times 10^{5}\right.$ cells/well in 6-well plates) were induced in four conditions for 21 days to chondrogenic differentiation evaluation, including commercial chondrogenic medium (control), 5\% PRP (HG-DMEM+5\% PRP), 10\% PRP (HG-DMEM+10\% PRP), and 15\% PRP (HG-DMEM+15\% PRP). The gene expression levels of ACAN, COL2A1, and SOX9 in pellets were detected. Morphological and pathological assessments were performed by the blind observer. After purifying, the percentages of cells with $\mathrm{CD} 105(+) / \mathrm{CD} 34(-)$ and $\mathrm{CD} 44(+) / \mathrm{CD} 45(-)$ were $96.5 \%$ and $92.9 \%$, respectively. The proliferation of BMSCs was enhanced in all groups, and 10\% PRP revealed more significant outcome than the others from day 5. The levels of ACAN, COL2A1, and SOX9 were lower in the three PRP groups than control group, but the levels of ACAN and SOX9 were higher in $10 \%$ PRP group than 5\% and 15\% PRP groups. Histological examinations showed that 10\% PRP-treated pellets had more regular appearance, larger size, and abundant extracellular matrix than $5 \%$ or $10 \%$ PRP groups, but still inferior to commercial chondrogenic medium. In conclusion, our results show that PRP may enhance the proliferation of rabbit BMSCs. However, PRP have limited effect on chondrogenic differentiation in comparison with commercial chondrogenic medium in pellets culture.
\end{abstract}

\section{Introduction}

Clinically, repairing massive articular cartilage defect remains a challenging issue. Current available surgical techniques include osteochondral transplantation and autologous chondrocyte implantation $[1,2]$. Despite of relative satisfying results, these techniques have been limited by morbidity of the donor sites and loss of the chondrogenic phenotype during in vitro expansion [3]. Alternatively, whether tissue-engineered cartilage can effectively repair massive articular cartilage defects has been a novel focus in this field.
Compared with chondrocytes, bone marrow-derived mesenchymal stem cells (BMSCs) have the potential for chondrogenic differentiation, self-renewal, and proliferation with less loss of phenotypes, which have been considered as an ideal cell source for tissue-engineered cartilage formation [4]. Previous studies have showed that BMSCs can be induced into hyaline-like cartilage tissue with high expression of aggrecan (ACAN), collagen type II (COL2), and SOX9 in high-density pellets or scaffold in vitro culture $[5,6]$. However, the extremely low percentage of BMSCs in bone marrow means that in vitro expansion is the first step to obtain enough cells 
before chondrogenic differentiation. Besides, the suitable medium containing growth factors is also an essential factor for tissue engineering. So far, the most widely applied mediums are almost commercially synthesized reagents, which have shown excellent chondrogenic-differentiation capacity in tissue-engineered cartilage formation [6-8]. While these medium contains kinds of ectogenic growth factors and are cost expensive, it is still worthy of further study whether some autologous biomaterials with similar induction effects can be an alternative to the synthetic reagents.

Platelet-rich plasma (PRP) is a plasma separated from autologous whole blood, with a high platelet proportion. After activation, PRP releases large amounts of growth factors, including transforming growth factor-beta (TGF- $\beta$ ), plateletderived growth factor (PDGF), insulin-like growth factor-1 (IGF-1), and vascular endothelial growth factor (VEGF). They can enhance tissue repair and regeneration. Clinically, PRP has been shown to inhibit inflammation, and induce cell proliferation and differentiation, resulting in pain relief and functional improvement in some musculoskeletal regenerated diseases [9-11]. Meanwhile, PRP plays an important role in the proliferation of chondrocytes and mesenchymal stem cells (MSCs) [12-15], and the induced chondrogenic differentiation of MSC in vitro culture [16-18]. However, limited studies have been reported on whether there are any differences in chondrogenic differentiation between autologous PRP and commercially synthesized medium, or whether PRP may replace these medium or not.

In the current study, we aimed to evaluate the effects of different concentrations of PRP on the proliferation and chondrogenic differentiation of BMSCs cultured in 3D pellets in vitro. We also determined if PRP alone was sufficient to induce the chondrogenesis of BMSCs in comparison with traditional chondrogenic differentiation medium.

\section{Materials and Methods}

2.1. Experimental Animals. Six mature male rabbits weighting $(1.75 \pm 0.25) \mathrm{kg}$ (Qinglong Mountain Experimental Animal Center, Nanjing, China) were used in this study. The animal experiments were approved by the Yangzhou University Medical School Animal Ethics Committee (YZMC/IACUC 2019101801, Yangzhou, China).

2.2. PRP Preparation. Under anesthetized condition, $50 \mathrm{ml}$ of whole blood was aspirated via heart puncture from each rabbit using $10 \mathrm{ml}$ vacutainer tubes containing $1 \mathrm{ml}$ sodium citrate (Nigale, Chengdu, China). PRP was prepared by a two-step method in room temperature according to our previous study [19]. Briefly, the first centrifugation was performed at $250 \times g$ for $7 \mathrm{~min}$; then, the upper plasma layer and middle buffy coat were gathered into another sterile coring tube to the second centrifugation at $350 \times \mathrm{g}$ for $10 \mathrm{~min}$. About $2 \mathrm{ml}$ of sediment and plasma were collected from the bottom to obtain PRP. All products were activated with one-tenth volume of $10 \% \mathrm{CaCl}_{2}$ solution and incubated overnight at $37^{\circ} \mathrm{C}$; then, they were centrifuged at $1000 \times g$ for $10 \mathrm{~min}$ to collect the supernatant to get activated PRP. The activated PRP samples were stored at $-70^{\circ} \mathrm{C}$ and used within one month for subsequent experiments.

2.3. Assay of Growth Factors and Cytokines. One milliliter of activated PRP and plasma of whole blood from each animal were used for the measurement of growth factors and cytokines. The levels of TGF- $\beta 3$, PDGF, IGF-1, and VEGF in samples were detected by enzyme-linked immunosorbent assay kits (Cusabio, Wuhan, China) with commercial kit (Nanjing Jiancheng Bioengineering Research Institute, Nanjing, China) according to manufacturer's instruction.

2.4. Isolation and Characterization of BMSCs. About $5 \mathrm{ml}$ bone marrow samples were obtained from each rabbit bilateral iliac crests and washed twice with high-glucose Dulbecco's modified Eagle's medium (HG-DMEM, Gibco, Waltham, MA, USA). They were resuspended in HG-DMEN with $10 \%(v / v)$ fetal bovine serum (FBS) (Gibco, Thermo Fisher Scientific, Waltham, MA, USA), $100 \mathrm{U} / \mathrm{ml}$ penicillin, and $0.1 \mathrm{mg} / \mathrm{ml}$ streptomycin. After 5 days of culture in an incubator with $5 \% \mathrm{CO}_{2}$ at $37^{\circ} \mathrm{C}$, nonadherent cells were removed; then, the medium was changed. Remaining adherent cells were further cultured until $80 \%$ confluence, then passaged at a ratio of $1: 3$ in new flasks. Culture medium was exchanged twice weekly. The phenotype of the passage 3 (P3) cells was characterized by detecting surface expression of CD34-phycoerythrin (PE) (GTX75414), CD45PE (GTX01462-08), CD44-fluorescein isothiocyanate (FITC) (GTX76381), and CD105-FITC (GTX11415) (Genetex, CA, USA) by flow cytometry according to manufacturer's instructions [20].

2.5. Cell Proliferation Assay. To observe cell proliferation in different medium, P3 BMSCs were enzymatically harvested and continued to culture in HG-DMEM $+10 \%$ FBS $(v / v)$, HG-DMEM + 5\% PRP $(v / v)$, HG-DMEM + 10\% PRP, and HG-DMEM + 15\% PRP, respectively. BMSCs were cultured in 6 -well culture plates $\left(5 \times 10^{3}\right.$ cells/ $100 \mu \mathrm{l} /$ well $)$, and the medium were changed every 3 days. Cell proliferation was assessed using a cell counting kit-8 (CCK8; Abcam, Cambridge, MA, USA) on days 1, 3, 5, and 7 of culture, as described previously [21].

2.6. BMSC Pellet Culture. Chondrogenesis was evaluated using high-density pellet cultures. P3 BMSCs $\left(5 \times 10^{5} /\right.$ well $)$ were pelleted in 6-well culture plates by centrifugation at $150 \times g$ for $5 \mathrm{~min}$ and then cultured in four different groups, including control (Ctrl), commercial chondrogenic medium (Cyagen, Guangzhou, China), 5\% PRP (HG-DMEM + 5\% PRP $(v / v)), 10 \%$ PRP (HG-DMEM + 10\% PRP), and 15\% PRP (HG-DMEM + 15\% PRP). Pellets were cultured for 21 days consecutively, and the culture medium was changed three times a week. Eight replicates were assigned for each group.

2.7. Reverse Transcription-Polymerase Chain Reaction (RTPCR) Analysis. BMSCs were harvested at day 21. From each group, 2 samples were homogenized in cold RIPA buffer (Beyotime Institute of Biotechnology, Shanghai, China), then extracted with total RNA extraction with the RNAiso Plus Kit (Takara, CA, USA). Complementary DNA (cDNA) 
was synthesized from RNA using the PrimeScript ${ }^{\mathrm{TM}}$ RT reagent Kit (Takara, CA, USA). The mRNA expression levels of aggrecan (ACAN), collagen type I (COL2A1), and SOX-9 were detected by RT-PCR, as described previously [22]. The primer sequences were designed and synthesized by General Biol (Hefei, Anhui, China) (Table 1). The products of RTPCR were quantitatively analyzed by the comparative CT method $\left({ }^{\triangle \Delta \mathrm{CT}}\right)$, and data were presented as target gene expression normalized to GAPDH (Sangon Biotech Co., Ltd., Shanghai, China) (Table 1).

2.8. Morphological Observation and Histological Examination. At the end of 3-week chondrogenic differentiation culture, followed by gross observation, all the pellets from the four groups were harvested and fixed in neutral-buffered formalin overnight at $4^{\circ} \mathrm{C}$. They were embedded in paraffin, and $5 \mu \mathrm{m}$ sections were cut and stained with standard toluidine blue (Bioscience \& Technology (Wuhan) Co., Ltd., Wuhan, China) to evaluate the production of extracellular matrix (ECM). Immunohistochemical staining was performed to detect COL2 expression (anti-COL2 antibody, rabbit polyclonal to COL22A1, Abcam, Cambridge, MA, USA; anti-GAPDH antibody, rabbit polyclonal antibody against GAPDH, Sangon Biotech Co., Ltd., Shanghai, China), according to manufacturer's instructions. All the sections were observed by a pathologist blindly. GAPDH was considered as the internal reference.

2.9. Statistical Analysis. All data were expressed as means \pm standard deviation (SD). Statistical analyses were performed using SPSS 18.0 (SPSS, IBM Software Inc., Armonk, $\mathrm{NY}$, USA). Independent-sample $t$-tests were used to evaluate the differences of growth factor levels in plasma and PRP. One-way analysis of variance (ANOVA) was used in the comparison of cell proliferation and gene expression levels, followed by the LSD test to assess the multiple comparisons. $P<0.05$ was considered statistically significant.

\section{Results}

3.1. Growth Factor Levels. The concentrations of TGF- $\beta 3$, PDGF, IGF-1, and VEGF were significantly increased in activated PRP compared with plasma (TGF- $\beta 3$ : $30.49 \pm$ 2.22 vs. $6.62 \pm 1.76 \mathrm{ng} / \mathrm{ml}$; PDGF: $1134.00 \pm 27.26$ vs. $371.42 \pm 56.24 \mathrm{pg} / \mathrm{ml}$; IGF- $1: 5.24 \pm 0.42$ vs. $2.72 \pm 0.32 \mathrm{ng} /$ $\mathrm{ml}$; and VEGF: $76.22 \pm 6.23$ vs. $28.10 \pm 6.05 \mathrm{pg} / \mathrm{ml}$, respectively, $P<0.05$, Figure 1 ).

3.2. Characteristics of Isolated BMSCs. After isolation and culture in vitro, P3 cell populations were characterized by flow cytometry to determine the expression of MSCassociated surface markers. The percentages of cells with CD105(+)/CD34(-) and CD44(+)/CD45(-) were $96.5 \%$ and $92.9 \%$, respectively (Figure 2 ).

3.3. BMSC Proliferation in PRP. The cell proliferation BMSCs in 10\% FBS, 5\% PRP, 10\% PRP, and 15\% PRP groups after $1,3,5$, and 7 days of culture were shown in Figure 3. Although proliferation increased with time was observed in all the groups, the effect was significantly weaker in the 5\% PRP group compared with the $10 \%$ FBS group
TABLE 1: Sequences of primers for RT-PCR analysis.

\begin{tabular}{lcc}
\hline Genes & Primer sequences & Product (bp) \\
\hline \multirow{2}{*}{ ACAN } & F-AACAGCCCAAGAAGCAGAA & 103 \\
& R-TGGGTCCAGAAATCCAGAATG & \\
COL2A1 & F-CAAGTCCCTCAACAACCAGAT & 124 \\
& R-TATCCAGTAGTCACCGCTCTT & \\
SOX9 & F-GGAGGAAGTCGGTGAAGAATG & \multirow{2}{*}{ R-TGCAGCGCCTTGAAGAT } \\
& F-AATCCACTGGCGTCTTCAC & 117 \\
GAPDH & R-TCACGCCCATCACAAACA & \\
&
\end{tabular}

$(P<0.05)$. BMSC proliferation was enhanced with the increasing PRP concentration, and stronger effects revealed in the $10 \%$ and $15 \%$ PRP groups compared with the $10 \%$ FBS group from day $5(P<0.05)$. Furthermore, proliferation assay appeared to be more obvious in 10\% PRP group than $15 \%$ PRP group from day 5 , though no significant difference was observed. Moreover, 7 days in vitro amplification results showed that compared with $10 \%$ FBS group, the proliferation rate in the 5\% PRP group was increased by 0.84 times, that in the 10\% PRP group was increased by 1.41 times, and that in the $15 \%$ PRP group was increased by 1.27 times $(P<0.05)$.

3.4. Expression of Cartilage-Specific Genes. The mRNA expression levels of ACAN, COL2A1, and SOX9 in cell pellets under the four different chondrogenic differentiation conditions after 21 days of culture were shown in Figure 4. The mRNA expression levels of ACAN and SOX9 were significantly higher in the $10 \%$ PRP group than the $5 \%$ and $15 \%$ groups, and COL2A1 mRNA expression was increased in $10 \%$ and $15 \%$ PRP groups compared with $5 \%$ PRP group $(P<0.05)$. However, their mRNA expression levels were all lower in PRP-treated groups compared with control group $(P<0.05)$.

3.5. Gross Morphology and Histological Examination. After 3-week in vitro culture, all the cell pellets revealed spheroid appearance, but with different sizes (Figure 5). Generally, the pellets in the control group revealed larger volume than that in the PRP-treated groups. However, the pellet sizes did not increase in line with PRP concentration. The diameter of pellets cultured in 15\% PRP was similar to 5\% PRP, and pellets exposed to 10\% PRP were obviously larger than those in 5\% and 15\% PRP groups, but still less than the control group.

Contents of cartilage-specific ECM, including ACAN and COL2, were staining with Safranin O, Alcian Blue, and immunohistochemistry (Figure 6). More abundant ACAN and COL2 depositions were observed in the control group than PRP-treated groups The pellets exposed to $10 \%$ PRP showed stronger ACAN and COL2 staining compared with the other two PRP groups, but still inferior to the control group. The enlarged image of immunohistochemical staining showed that there were more chondrocytes in the control group, and there was COL2 positive staining around 

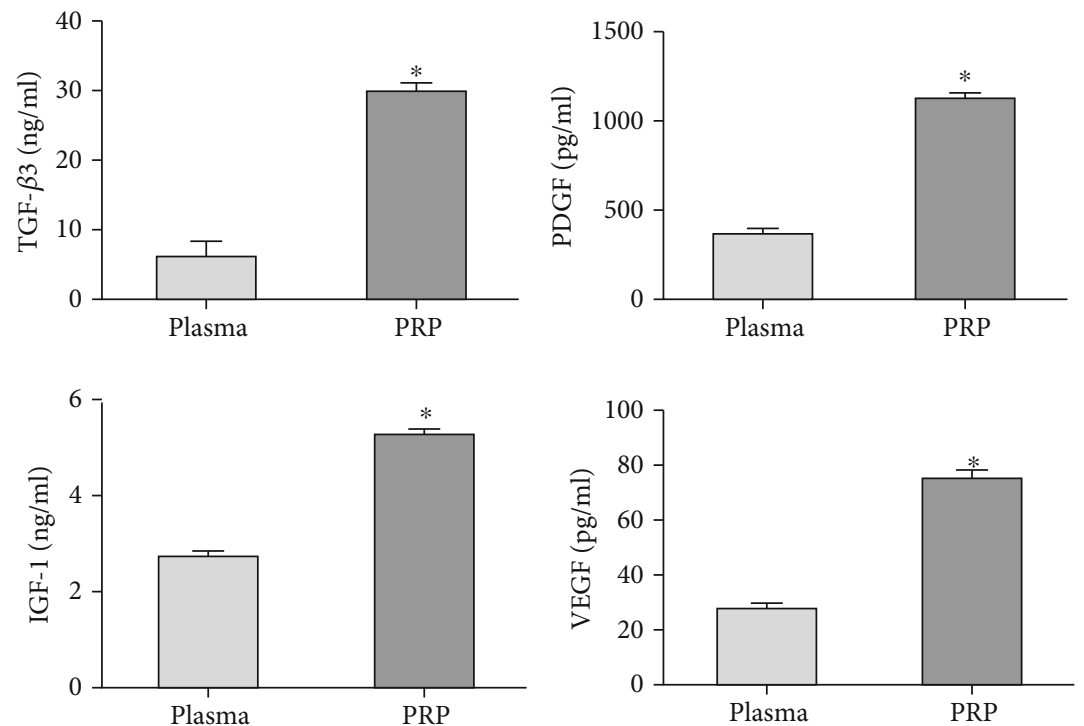

Figure 1: Growth factor levels in plasma and activated PRP. Concentrations of TGF- $\beta 3$, PDGF, IGF-1, and VEGF were significantly increased in PRP compared with plasma $\left({ }^{*} P<0.05, n=6\right)$.

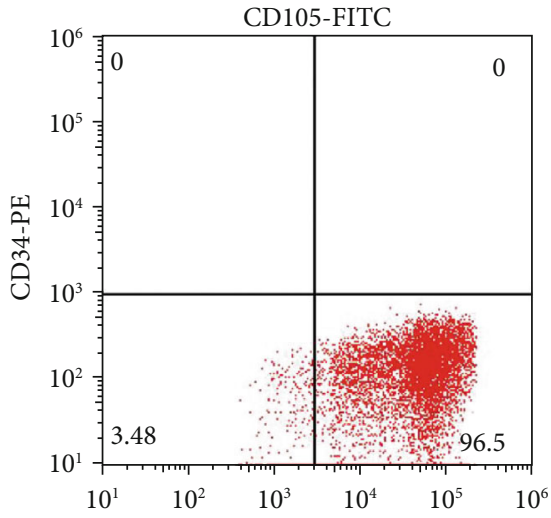

(a)

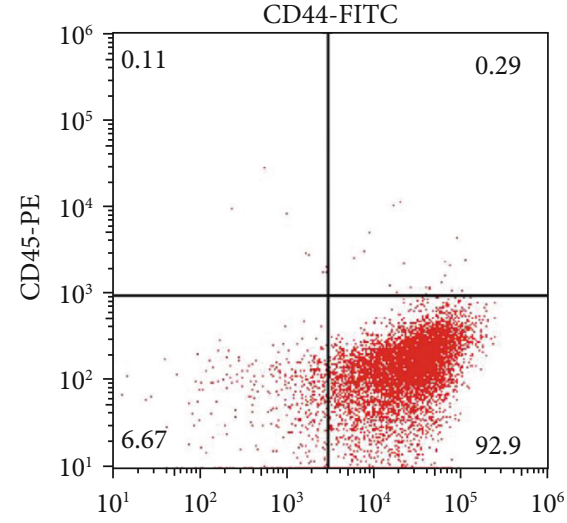

(b)

Figure 2: Surface marker profiles of isolated and cultured BMSCs. The percentages of CD105+/CD34 (a) and CD44+/CD45 (b) cells were $96.5 \%$ and $92.9 \%$, respectively.

them, followed by $10 \%$ PRP group and 15\% PRP group. The content of chondrocytes and COL2 in 5\% PRP group was the least (Figure 7).

\section{Discussion}

PRP is an autologous blood product containing a variety of bioactive components with anti-inflammatory, tissue repair, and regeneration-promoting functions, which has been widely used in the treatment of chronic degenerative diseases [9-11]. In vitro experiments have confirmed the ability of PRP to enhance the proliferation of chondrocytes and MSCs [12-15]. However, the effect of PRP on cartilage differentiation of BMSCs in tissue engineering remains controversial. Although recent studies $[17,18,23]$ have demonstrated the effects of different concentrations of PRP on chondrogenic dif- ferentiation of MSCs, they do not compare PRP with traditional commercial cartilage induction medium. Therefore, we aimed to compare the effects on cartilage differentiation of BMSCs between different PRP concentrations and commercial cartilage induction medium and to evaluate the role of PRP in tissue-engineered cartilage formation.

In this study, the concentrations of TGF- $\beta 3$, IGF- 1 , PDGF, and VEGF were significantly increased in activated PRP compared with plasma. The addition of high concentrations of PRP to basic medium promoted the expansion of BMSCs in vitro compared with $10 \%$ FBS, revealing a concentration time-dependent effect. Higher concentrations of growth factors and cytokines in PRP may induce this result.

Commercial MSC chondrogenic differentiation media from different manufacturers have been widely used in chondrogenic differentiation experiments. These synthetic 

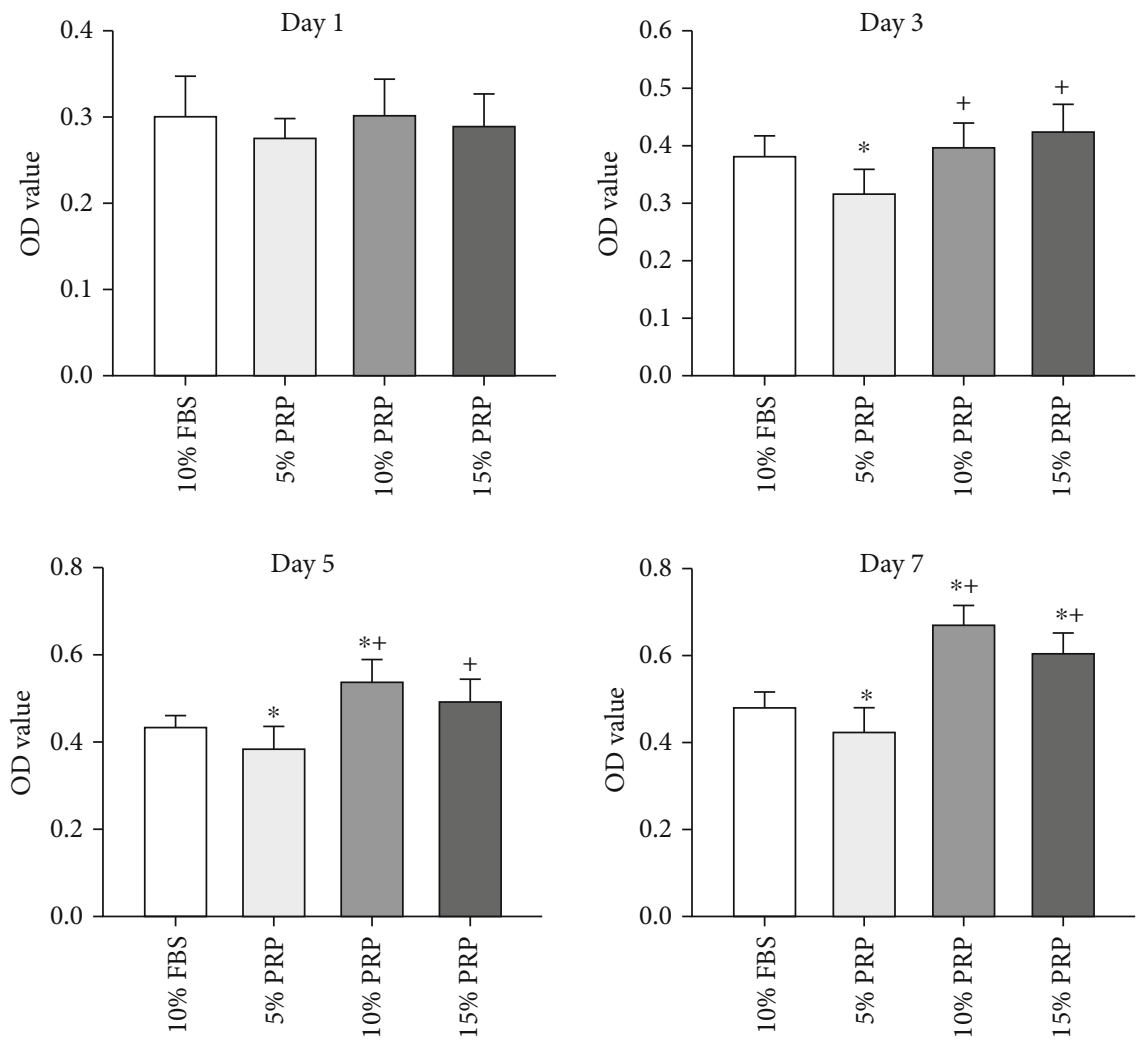

FIGURE 3: Cell counting assay for BMSC proliferation in the different medium. ${ }^{*} P<0.05$ compared to $10 \%$ FBS group, ${ }^{+} P<0.05$ compared to 5\% PRP group. OD: optical density; FBS: fetal bovine serum; PRP: platelet-rich plasma.
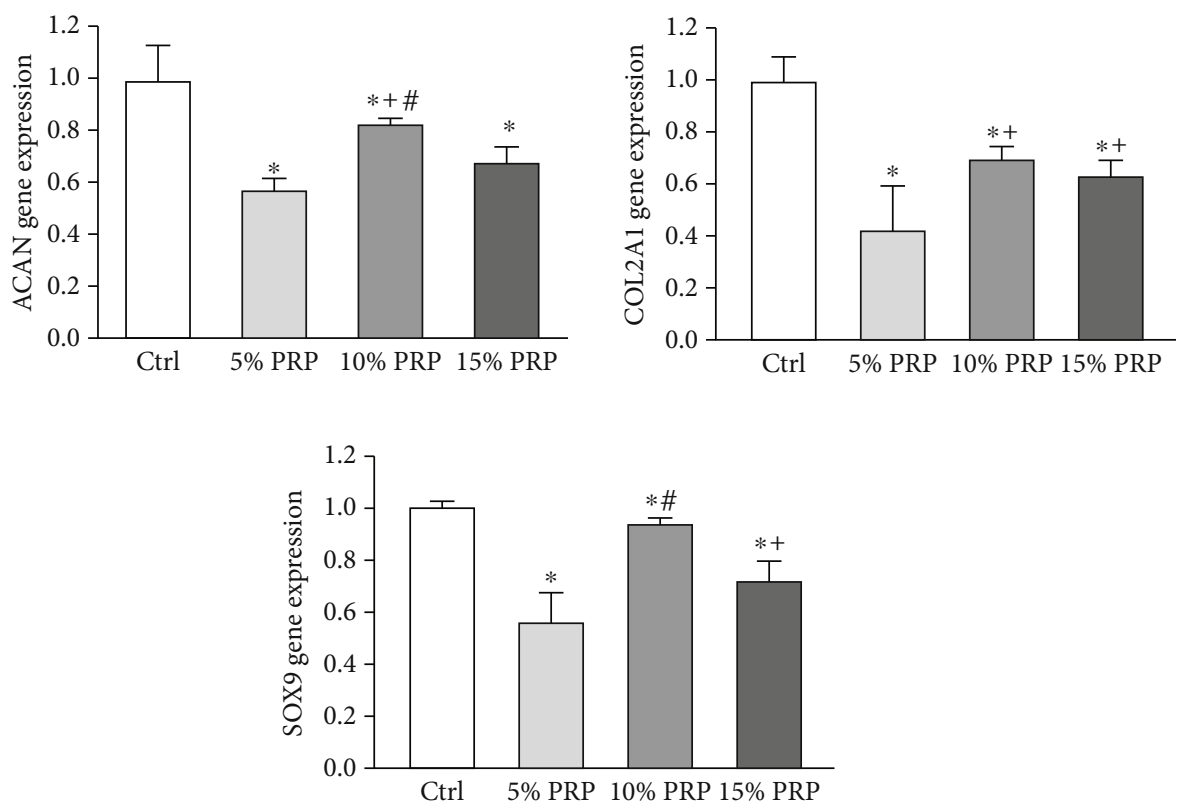

Figure 4: Expression of cartilage-specific genes in the four groups following 21-day culture. Ctrl: commercial chondrogenic differentiation medium. ${ }^{*} P<0.05$ compared to control group, ${ }^{+} P<0.05$ compared to $5 \%$ PRP group, ${ }^{\#} P<0.05$ compared to $15 \%$ PRP group.

reagents contain not only basic cell culture medium but also additional ingredients, such as TGF- $\beta 3$, insulin, transferrin and selenium supplement, ascorbate, sodium pyruvate, pro- line, and dexamethasone. Among these, TGF- $\beta 3$ is a vital regulatory factor in promoting chondrogenic differentiation of MSCs, while the other components also improve cell 

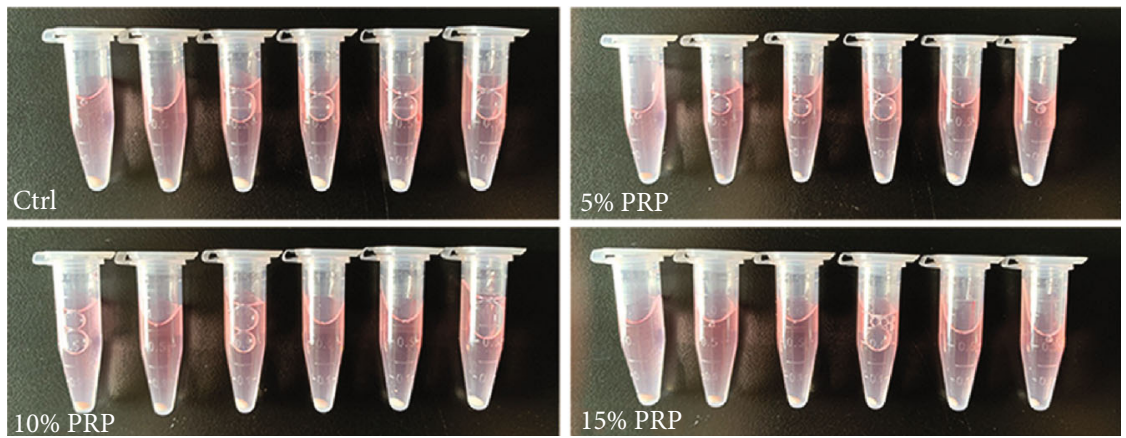

FIGURE 5: Gross morphology of BMSC pellets in four different media after 3-week culture in vitro.

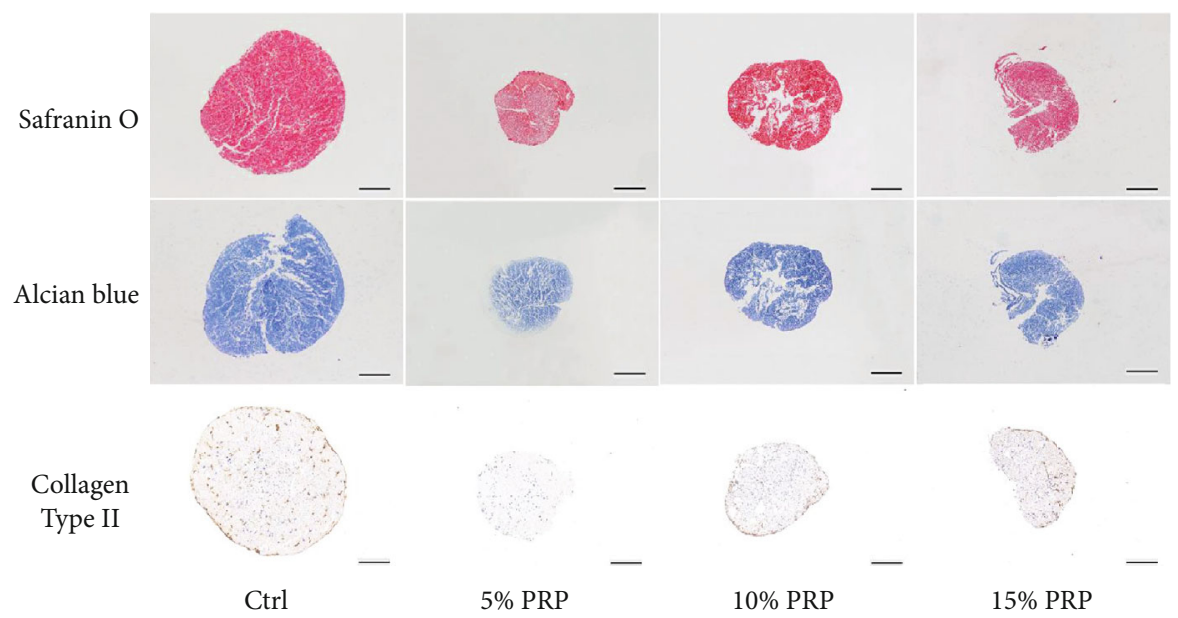

FiguRE 6: Histological evaluation of aggrecan and collagen type II deposition in BMSC pellets from four different groups after 3-week culture in vitro. Scale bar $=500 \mu \mathrm{m}$.

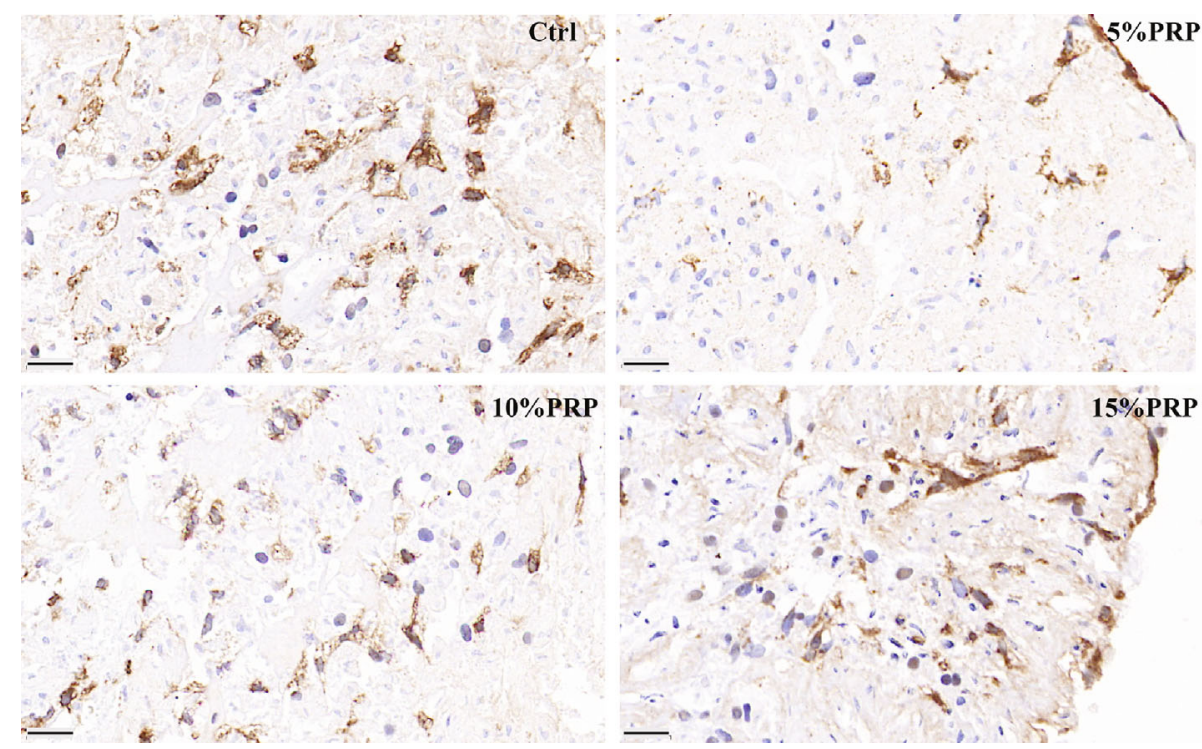

FIGURE 7: Type II collagen immunohistochemistry of BMSC pellets after 3-week culture in vitro in commercial chondrogenic medium (Ctrl) and 3 PRP groups. Positive staining is indicated by a reddish brown color. Scale bar $=20 \mu \mathrm{m}$. 
metabolism, promote cell proliferation, and inhibit cell aging. The concentration of TGF- $\beta 3$ in the cartilage differentiation medium used in this study was $10 \mathrm{ng} / \mathrm{ml}$, which was higher than that in 10\% PRP $(3.05 \mathrm{ng} / \mathrm{ml})$, while PRP itself did not contain any other additives. Therefore, we considered that basic cell culture medium with $10 \%$ PRP might have a limited effect on BMSC cartilage differentiation compared with commercial cartilage differentiation medium.

It is not clear that whether the increased PRP concentration in medium would enhance chondrogenic differentiation in BMSCs. Krüger et al. have reported that human PRP enhances the migration and stimulates the chondrogenic differentiation of human BMSCs derived from spongious bone of the tibia or femur head [16]. Liou et al. have investigated the effects of different concentrations of PRP on adiposederived stem cells, and they have shown that increasing the PRP concentration do not enhance chondrogenic differentiation [18]. Amaral et al. also have observed cartilage differentiation medium containing different PRP concentrations on chondrogenesis of BMSC pellets, and they find that increasing the PRP proportion from $1 \%$ to $10 \%$ reduces the expression levels of cartilage-specific genes and proteoglycans in pellets [23]. In addition, some previous studies have reported that commercial induction medium containing 10\% PRP do not enhance chondrogenic differentiation compared with induction medium alone [17]. The similar results are also observed in this study. Though certain PRP concentration induces chondrogenic differentiation in BMSCs pellets, this effect is not enhanced with the increasing of PRP contents.

In this study, we observed that 10\% PRP promoted the chondrogenic differentiation of BMSCs pellets by analysis of gene expression levels, gross morphological observation, and immunohistochemical staining of cartilage-specific proteins. We supposed that the biological mechanism of PRP in chondrogenic differentiation of MSCs may be complicated and intricate. Furthermore, it needs further study to observe whether MSC derived from different sources respond consistently to PRP in induction chondrogenesis.

Activated-PRP includes cytokines, such as TGF $\beta$, PDGF, VEGF, and epidermal growth factor (EGF), but the beneficial effects of these growth factors in chondrogenesis remain unclear. The TGF $\beta$ family is known to induce proliferation and chondrogenesis of BMSCs during cartilage formation. Meanwhile, PDGF supports chondrocytes to maintain the hyaline-like chondrogenic phenotype and induces proteoglycan synthesis [24]. However, VEGF shows poor chondrogenic effects on muscle-derived stem cells in a rat model [25], and an EGF receptor ligand promotes chondrocyte catabolic activity and inhibits anabolic activity in an osteoarthritis mouse model [26]. VEGF and/or EGF may therefore weaken the chondro-inductive effects of PRP. Further studies are needed to determine if specific depletion of such antichondrogenic factors and optimization of other stimulatory components in PRP might enhance BMSC chondrogenic differentiation.

In addition to optimizing the differentiation induction medium, it is also necessary to improve the seed cells to facilitate the construction of tissue-engineered cartilage. Some researchers have constructed a coculture system com- posed of autologous chondrocytes and MSCs in order to observe the efficiency of cartilage differentiation and to inhibit chondrocyte hypertrophy and osteogenic differentiation by the paracrine effect of different cells [27, 28]. Although the results were not consistent, they provided a novel research direction for the construction of tissue-engineered cartilage. Recent studies have reported that human placental [29], umbilical cord blood [30], and amnion-derived MSCs [31] reveal high proliferative ability, multipotency, and low immunogenicity, which become novel seed cells in tissue engineering. Further studies are needed to observe whether PRP could enhance chondrogenic differentiation in these stem cells or not.

There are also some limitations in this study. Firstly, we only observed the end-stage chondrogenic differentiation of BMSCs after 21 days of culture, and the continuous effect of PRP were not examined. Secondly, limited number of PRPtreated groups were designed; the details of concentrationdependent effect of PRP on chondrogenesis were not enough. Additionally, due to commercial chondrogenic differentiation media from different manufacturers may contain various components and efficacy, the reagents used in this experiment are of limited representativeness.

\section{Conclusion}

Activated-PRP contains abundant growth factors and cytokines, which can enhance BMSC proliferation in vitro and induce chondrogenic differentiation in $3 \mathrm{D}$ pellet culture. However, the effects of PRP alone used were limited compared with traditional commercial MSC chondrogenic differentiation media. Future studies that aim at optimizing and modifying the composition and concentration of PRP may have satisfying results in tissue-engineered cartilage formation.

\section{Data Availability}

The datasets used in the current study are available from the corresponding author on reasonable request by email.

\section{Conflicts of Interest}

All the authors declare that they have no interest conflicts.

\section{Acknowledgments}

The authors thank the support from Junzheng Hu M.D. and Ming Li M.M. from the Yangzhou University Medical School in flow cytometry and histological analysis. The authors would like to thank International Science Editing (http://www.internationalscienceediting.com) for language editing of this manuscript. The authors also thank Research Square (https://www.researchsquare.com/) for preprint service of this manuscript. This work was supported by the Military Medical Scientific Research Foundation of China (grant number 16QNP044), Nanjing Medical Science and Technique Development Foundation of China (grant numbers YKK18223 and YKK19142), and National Natural Science Foundation of China (grant number 81902197). 


\section{References}

[1] B. R. Mandelbaum, J. E. Browne, and F. Freddie, "Articular cartilage lesions of the knee," The American Journal of Sports Medicine, vol. 26, no. 6, pp. 853-861, 1998.

[2] P. Visña, L. Pasa, I. Cizmár, R. Hart, and J. Hoch, “Treatment of deep cartilage defects of the knee using autologous chondrograft transplantation and by abrasive techniques-a randomized controlled study," Acta Chirurgica Belgica, vol. 104, no. 6, pp. 709-714, 2004.

[3] C. Chan, C. Richmond, K. G. Shea, and S. L. Frick, "Management of osteochondritis dissecans of the femoral condyle," JBJS Rev, vol. 6, no. 3, article e5, 2018.

[4] S. Giovannini, J. Diaz-Romero, T. Aigner, P. Heini, P. MainilVarlet, and D. Nesic, "Micromass co-culture of human articular chondrocytes and human bone marrow mesenchymal stem cells to investigate stable neocartilage tissue formation in vitro," European Cells \& Materials, vol. 20, pp. 245-259, 2010.

[5] Z. Li, R. Ba, Z. Wang, J. Wei, Y. Zhao, and W. Wu, "Angiogenic potential of human bone marrow-derived mesenchymal stem cells in chondrocyte brick-enriched constructs promoted stable regeneration of craniofacial cartilage," Stem Cells Translational Medicine, vol. 6, no. 2, pp. 601-612, 2017.

[6] S. Sulaiman, S. R. Chowdhury, M. B. Fauzi et al., "3D culture of MSCs on a gelatin microsphere in a dynamic culture system enhances chondrogenesis," International Journal of Molecular Sciences, vol. 21, no. 8, p. 2688, 2020.

[7] X. Li, M. Wang, X. Jing et al., "Bone marrow- and adipose tissue-derived Mesenchymal stem cells: characterization, differentiation, and applications in cartilage tissue engineering," Critical Reviews in Eukaryotic Gene Expression, vol. 28, no. 4, pp. 285-310, 2018.

[8] X. Ouyang, Y. Xie, and G. Wang, "Mechanical stimulation promotes the proliferation and the cartilage phenotype of mesenchymal stem cells and chondrocytes co-cultured_in vitro_," Biomedicine \& Pharmacotherapy, vol. 117, p. 109146, 2019.

[9] J. W. Hammond, R. Y. Hinton, and C. L. Ann, "Use of autologous platelet-rich plasma to treat muscle strain injuries," The American Journal of Sports Medicine, vol. 37, no. 6, pp. 1135-1142, 2009.

[10] A. J. Hernandez, A. Pedrinelli, A. M. DeAlmeida, M. K. Demange, M. F. Sobrado, and M. B. Rodrigues, "Patellar tendon healing with platelet-rich plasma: a prospective randomized controlled trial," The American Journal of Sports Medicine, vol. 40, no. 6, pp. 1282-1288, 2012.

[11] P. Sandeep, M. S. Dhillon, and B. Tungish, "Randomized controlled trial comparing hyaluronic acid, platelet-rich plasma and the combination of both in the treatment of mild and moderate osteoarthritis of the knee- letter to the editor \& author response," Journal of Stem Cells and Regenerative Medicine, vol. 13, no. 2, pp. 80-83, 2017.

[12] K. Akeda, H. S. An, M. Okuma et al., "Platelet-rich plasma stimulates porcine articular chondrocyte proliferation and matrix biosynthesis," Osteoarthritis and Cartilage, vol. 14, no. 12, pp. 1272-1280, 2006.

[13] V. D'Esposito, F. Passaretti, G. Perruolo et al., "Platelet-rich plasma increases growth and motility of adipose tissuederived mesenchymal stem cells and controls adipocyte secretory function," Journal of Cellular Biochemistry, vol. 116, no. 10, pp. 2408-2418, 2015.
[14] V. Jeyakumar, E. Niculescu-Morzsa, C. Bauer, Z. Lacza, and S. Nehrer, "Platelet-rich plasma supports proliferation and redifferentiation of chondrocytes during in vitro expansion," Frontiers in Bioengineering and Biotechnology, vol. 5, p. 75, 2017.

[15] A. R. Hoberman, C. Cirino, M. B. McCarthy et al., "Bone marrow-derived mesenchymal stromal cells enhanced by platelet-rich plasma maintain adhesion to scaffolds in arthroscopic simulation," Arthroscopy, vol. 34, no. 3, pp. 872-881, 2018.

[16] J. P. Krüger, S. Hondke, M. Endres, A. Pruss, A. Siclari, and C. Kaps, "Human platelet-rich plasma stimulates migration and chondrogenic differentiation of human subchondral progenitor cells," Journal of Orthopaedic Research, vol. 30, no. 6, pp. 845-852, 2012.

[17] J. K. Lee, S. Lee, S. A. Han, S. C. Seong, and M. C. Lee, “The effect of platelet-rich plasma on the differentiation of synovium-derived mesenchymal stem cells," Journal of Orthopaedic Research, vol. 32, no. 10, pp. 1317-1325, 2014.

[18] J. J. Liou, B. B. Rothrauff, P. G. Alexander, and R. S. Tuan, "Effect of platelet-rich plasma on chondrogenic differentiation of adipose- and bone marrow-derived mesenchymal stem cells," Tissue Engineering. Part A, vol. 24, no. 19-20, pp. 1432-1443, 2018.

[19] Z. Wang, C. Zhai, H. Fei et al., "Intraarticular injection autologous platelet-rich plasma and bone marrow concentrate in a goat osteoarthritis model," Journal of Orthopaedic Research, vol. 36, no. 8, pp. 2140-2146, 2018.

[20] I. Hussain, S. A. Magd, O. Eremin, and M. El-Sheemy, "New approach to isolate mesenchymal stem cell (MSC) from human umbilical cord blood," Cell Biology International, vol. 36, no. 7, pp. 595-600, 2012.

[21] A. Mishra, P. Tummala, A. King et al., "Buffered platelet-rich plasma enhances mesenchymal stem cell proliferation and chondrogenic differentiation," Tissue Engineering. Part C, Methods, vol. 15, no. 3, pp. 431-435, 2009.

[22] J. Shen, Q. Gao, Y. Zhang, and Y. He, “Autologous platelet-rich plasma promotes proliferation and chondrogenic differentiation of adipose-derived stem cells," Molecular Medicine Reports, vol. 11, no. 2, pp. 1298-1303, 2015.

[23] R. J. F. C. do Amaral, A. Matsiko, M. R. P. Tomazette et al., "Platelet-rich plasma releasate differently stimulates cellular commitment toward the chondrogenic lineage according to concentration," Journal of Tissue Engineering, vol. 6, 2015.

[24] E. Kon, R. Buda, G. Filardo et al., "Platelet-rich plasma: intraarticular knee injections produced favorable results on degenerative cartilage lesions," Knee Surgery, Sports Traumatology, Arthroscopy, vol. 18, no. 4, pp. 472-479, 2010.

[25] T. Matsumoto, G. M. Cooper, B. Gharaibeh et al., "Cartilage repair in a rat model of osteoarthritis through intraarticular transplantation of muscle-derived stem cells expressing bone morphogenetic protein 4 and soluble Flt-1," Arthritis and Rheumatism, vol. 60, no. 5, pp. 1390-1405, 2009.

[26] D. L. Long, V. Ulici, S. Chubinskaya, and R. F. Loeser, "Heparin-binding epidermal growth factor-like growth factor (HBEGF) is increased in osteoarthritis and regulates chondrocyte catabolic and anabolic activities," Osteoarthritis and Cartilage, vol. 23, no. 9, pp. 1523-1531, 2015.

[27] R. Ramezanifard, M. Kabiri, and A. H. Hanaee, "Effects of platelet rich plasma and chondrocyte co-culture on MSC chondrogenesis, hypertrophy and pathological responses," EXCLI Journal, vol. 16, pp. 1031-1045, 2017. 
[28] M. M. Pleumeekers, L. Nimeskern, J. Koevoet, M. Karperien, K. S. Stok, and G. van Osch, "Trophic effects of adiposetissue-derived and bone-marrow-derived mesenchymal stem cells enhance cartilage generation by chondrocytes in co-culture," PLoS One, vol. 13, no. 2, article e0190744, 2018.

[29] Y. Park, S. Seo, J. Kim, J. Heo, Y. Lim, and C. Ha, "Effect of chondrocyte-derived early extracellular matrix on chondrogenesis of placenta-derived mesenchymal stem cells," Biomedical Materials, vol. 10, no. 3, 2015.

[30] R. Rakic, B. Bourdon, M. Demoor, S. Maddens, N. Saulnier, and P. Galéra, "Differences in the intrinsic chondrogenic potential of equine umbilical cord matrix and cord blood mesenchymal stromal/stem cells for cartilage regeneration," Scientific Reports, vol. 8, no. 1, 2018.

[31] M. Nogami, H. Tsuno, C. Koike et al., "Isolation and characterization of human amniotic mesenchymal stem cells and their chondrogenic differentiation," Transplantation Journal, vol. 93, no. 12, pp. 1221-1228, 2012. 